\title{
CARACTERIZAÇÃO DA INFRAESTRUTURA E DO PROCESSO DE TRABALHO NA ASSISTÊNCIA AO PRÉ-NATAL*
}

\author{
Cintia Mikaelle Cunha de Santiago Nogueira ${ }^{1}$, Jéssica Micaele Rebouças Justino², Maria Isabel Paiva \\ Linhares'Tavares ${ }^{2}$, Fátima Raquel Rosado Morais ${ }^{3}$
}

\begin{abstract}
RESUMO: Pesquisa descritiva exploratória com enfoque quantitativo, realizada entre os meses de novembro de 2014 e março de 2015, envolvendo 25 Unidades Básicas de Saúde com Estratégia Saúde da Família, 35 profissionais que assistem ao pré-natal e 305 usuárias da atenção básica de um município do Rio Grande do Norte, para caracterizar a infraestrutura e o processo de trabalho nos serviços de atenção ao pré-natal de baixo risco. Evidenciou-se que, no geral, a infraestrutura foi considerada adequada. Na variável processo de trabalho, foram detectadas $24(68,6 \%)$ unidades com abordagem multiprofissional, com realização de todos os procedimentos clínico-obstétricos (100\%). No cotidiano assistencial, $186(61 \%)$ gestantes relataram que há atividades de educação em saúde, e, para $22(62,9 \%)$ profissionais, a visita puerperal foi considerada adequada. Estudos como estes podem ser elaborados e ações implementadas, no intuito de propiciar o melhoramento da assistência realizada durante o pré-natal.
\end{abstract}

DESCRITORES: Cuidado pré-natal; Assistência à saúde; Avaliação em saúde; Infraestrutura sanitária; Estratégia Saúde da Família.

\section{CHARACTERIZATION OF THE STRUCTURE AND WORK PROCESS IN PRENATAL CARE}

ABSTRACT: Descriptive, exploratory study with a quantitative approach conducted between November 2014 and March 2015, involving 25 Basic Health Units (UBS) that implement the Family Health Strategy, 35 professionals who provide prenatal care and 305 users of basic care services of one city of Rio Grande do Norte, aimed to characterize the structure and work process in low-risk prenatal care services. In general, structure was considered adequate. Regarding the variable work process, 24 (68.6\%) units with a multidisciplinary approach that perform all clinical-obstetric procedures were detected (100\%). Regarding daily care activities, 186 $(61 \%)$ pregnant women reported the existence of health education activities, and $22(62.9 \%)$ health professionals described postnatal visits as adequate. Further studies on the topic and implementation of actions aimed to improve prenatal care are recommended. DESCRIPTORS: Prenatal care; Health care; Health assessment; Health structure; Family Health Strategy.

\section{CARACTERIZACIÓN DE LA INFRAESTRUCTURA Y DEL PROCESO DE TRABAJO EN LA ATENCIÓN DEL PRENATAL}

RESUMEN: Investigación descriptiva, exploratoria, con enfoque cuantitativo, realizada entre noviembre de 2014 y marzo de 2015 , involucrando 25 Unidades Básicas de Salud con Estrategia Salud de la Familia, 35 profesionales que atienden el prenatal y 305 usuarias de atención básica en municipio de Rio Grande do Norte, para caracterizar la infraestructura y el proceso de trabajo en servicios de atención del prenatal de bajo riesgo. Se evidenció en general que la infraestructura fue considerada adecuada. En la variable proceso de trabajo, fueron detectadas $24(68,6 \%)$ unidades con abordaje multiprofesional, con realización de todos los procedimientos clínico-obstétricos (100\%). En la atención del día a día, 186 (61\%) embarazadas informaron existencia de actividades de educación en salud y, para $22(62,9 \%)$ profesionales, la visita puerperal fue considerada adecuada. Estudios como este pueden llevarse a cabo, e implementarse acciones con la intención de favorecer la mejora de la atención brindada durante el prenatal.

DESCRIPTORES: Atención Prenatal; Prestación de Atención de Salud; Evaluación en Salud; Infraestructura Sanitaria; Estrategia de Salud Familiar.

\footnotetext{
*Artigo extraído da dissertação intitulada: “Avaliação da assistência de pré-natal de risco habitual no município de MossoróRN". Universidade do Estado do Rio Grande do Norte, 2016.
}

${ }^{1}$ Enfermeira. Mestre em Saúde e Sociedade. Doutoranda em Bioquímica e Fisiologia pela Universidade Federal de Pernambuco. Recife, PE, Brasil.

${ }^{2}$ Discente de Enfermagem. Universidade do Estado do Rio Grande do Norte. Mossoró, RN, Brasil.

${ }^{3}$ Enfermeira. Doutora em Psicologia Social. Docente de Enfermagem da Universidade do Estado do Rio Grande do Norte. Mossoró, RN, Brasil.

Autor Correspondente:

Jéssica Micaele Rebouças Justino

Universidade do Estado do Rio Grande do Norte

R. Desembargador Dionísio Filgueira, 376 - 59610-090 - Mossoró, RN, Brasil

Email: jessicareboucaas@gmail.com
Recebido: $15 / 03 / 2016$ Finalizado: 07/11/2016 


\section{- INTRODUÇÃO}

A gestação consiste de um processo fisiológico natural e compreende uma sequência de alterações físicas, psicológicas e sociais específicas, que demanda adaptações no corpo e na vida da mulher ${ }^{(1)}$. Na maioria das vezes a gestação e o parto evoluem sem intercorrências, embora, em algumas situações, a mulher desenvolva doenças, agravos ou problemas de saúde que aumentem a probabilidade de um desfecho desfavorável para o feto e/ou para $\mathrm{si}^{(2)}$.

Na assistência pré-natal é realizado o monitoramento das principais intercorrências gestacionais, buscando evitar complicações e elevação na mortalidade materna e neonatal ${ }^{(3)}$. Todavia, em 2013, foi anunciado o acontecimento de mais de 300 mil mortes maternas em todo o mundo, a maioria ocorrendo nos países em desenvolvimento ${ }^{(4)}$.

No Brasil, a morte materna se apresenta como um grave problema de saúde pública, e, os seus maiores percentuais são encontrados na região Nordeste do país ${ }^{(5)}$. É ainda mais preocupante saber que geralmente seriam mortes evitáveis e que atingem, mais frequentemente, populações com menor acesso a bens sociais e a serviços qualificados de saúde em tempo oportuno ${ }^{(6)}$.

De fato, o acompanhamento pré-natal é fundamental para detecção precoce de intercorrências para o tratamento adequado com a otimização de resultados de saúde materna e infantil(7). Para potencializar essa dimensão deve se estabelecer parâmetros de avaliação da qualidade da assistência ao processo gestacional, o que proporcionaria melhor entendimento dos aspectos que interatuam e problematizam essa dinâmica.

Pensando a avaliação, desde a década de 1970, inúmeros instrumentos foram propostos e alguns buscavam classificar a adequação dos cuidados de pré-natal. Na atualidade, a avaliação da qualidade do pré-natal utiliza parâmetros quantitativos como número de consultas e a idade gestacional de ingresso no serviço de saúde ${ }^{(8)}$.

No entanto, havia a necessidade de se avaliar não apenas os aspectos quantitativos do processo, mas enveredar pela dimensão subjetiva desse cuidado. Nessa perspectiva, foi proposto o Índice Infraestrutura, Processo de trabalho e Resultados no Pré-natal (IPR/PN), baseado nas diretrizes do Programa de Humanização do Pré-natal e do Nascimento (PHPN) ${ }^{(9-10)}$ e que insere elementos quantitativos e qualitativos, distribuídos na tríade infraestrutura, processo de trabalho e resultados. Essa tríade baseia-se na proposta de avaliação de qualidade de atenção de Donabedian ${ }^{(11)}$.

Focando a infraestrutura, é possível enfatizá-la como indispensável para um adequado pré-natal, pois o profissional precisa de recursos materiais e de insumos que possibilitem a realização de ações assistenciais completas e constantes. Outra dimensão, que é o processo de trabalho, avalia a relação direta entre profissionais e gestantes. A partir dessa interação são detectadas possíveis intercorrências clínicas e identificados anseios e necessidades que permeiam todo o processo gravídico vivenciado por essa mulher.

Pela importância da infraestrutura e do processo de trabalho na qualificação do acompanhamento pré-natal, este estudo objetiva caracterizar a infraestrutura e o processo de trabalho nos serviços de atenção ao pré-natal de baixo risco.

\section{MÉTODO}

Pesquisa descritiva exploratória, com enfoque quantitativo e recorte de pesquisa de mestrado intitulada "Avaliação da assistência de pré-natal de risco habitual no município de Mossoró-RN".

Foi desenvolvida com profissionais de saúde e usuárias que realizam o pré-natal no município de Mossoró-RN, cujo espaço da investigação abrangeu as Unidades Básicas de Saúde (UBS) com Estratégia Saúde da Família (ESF)de Mossoró-RN e Unidades que tinham a atuação de equipes de saúde da família. Após essa delimitação, foram incluídas 25 (vinte e cinco) unidades com ESF.

Na definição da amostra dos profissionais, foram incluídos os que realizavam o acompanhamento pré-natal nas unidades investigadas e excluídos os que estavam em gozo de férias, licença ou atestado 
médico. Na amostra das usuárias das Unidades Básicas de Saúde com ESF investigadas foram incluídas mulheres maiores que 18 anos, que estavam em acompanhamento pré-natal ou que tinham crianças com até seis meses de vida. Foram excluídas as gestantes que tinham algum tipo de transtorno mental.

Nas áreas investigadas havia um total de 46 (quarenta e seis) profissionais atuando nas equipes de saúde da família das 25 (vinte e cinco) unidades que fazem parte dessa pesquisa e, após aplicação dos critérios de inclusão e exclusão, a amostra contemplou 35 (trinta e cinco) profissionais, médicos ou enfermeiros atuantes na assistência de pré-natal que ao atender os critérios de inclusão e exclusão estavam aptos a responder o componente do processo de trabalho.

Já o questionário referente aos dados da infraestrutura da UBS demanda a resposta de um único profissional da unidade, com isso, foi aplicado como critério de inclusão, o profissional que atuava a mais tempo no serviço, participaram dessa etapa da pesquisa o total de 25 (vinte e cinco) profissionais. Para o cálculo da amostra das usuárias, utilizou-se a fórmula para populações finitas e considerou-se a média de gestantes em acompanhamento pré-natal, no ano de 2013, nas unidades investigadas. Ao final, a amostra ficou composta por 305 usuárias. Essa amostra foi captada de maneira espontânea, os pesquisadores se dirigiram as UBS em dias de atendimento e convidavam as usuárias que atendiam os critérios de inclusão e exclusão a participar da pesquisam, após assinatura do Termo de Consentimento Livre e Esclarecido.

A coleta dos dados aconteceu entre os meses de novembro de 2014 e março de 2015, a partir do instrumento denominado de índice IPR/PRÉ-NATAL, construído com base nas diretrizes do Programa de Humanização de Pré-Natal e Nascimento do Ministério da Saúde, durante uma pesquisa de mestrado em ciências da nutrição da Universidade Federal da Paraíba (UFPB) ${ }^{(10)}$.

As variáveis relacionadas as ações realizadas no acompanhamento pré-natal foram determinadas como adequadas quando a atividade foi realizada com mais de $90 \%$ das usuárias em acompanhamento pré-natal e inadequadas quando as atividades atingiam menos de $90 \%$ das usuárias.

Os dados coletados foram organizados em tabelas e analisados a partir de autores que abordam a temática e que foram utilizados nessa investigação.A análise estatística foi realizada por meio do programa estatístico IBM SPSS Statistics for Windows versão 22.0. Para evidenciar diferenças nas proporções nas variáveis estudadas, utilizou-se o teste de Qui-quadrado para proporções homogêneas. Valores de $p<0,05$ foram considerados significativos.

A investigação foi submetida ao Comitê de Ética em Pesquisa da Universidade do Estado do Rio Grande do Norte e aprovado em 27 de outubro de 2014, sob o parecer ${ }^{\circ} 854.846$.

\section{RESULTADOS}

O levantamento do perfil socioeconômico das usuárias acompanhadas na consulta de pré-natal demonstrou que a renda familiar de 202 (66,2\%) gestantes variou entre 1 e 2 salários mínimos; 190 $(62,3 \%)$ gestantes na faixa etária compreendida entre 18 e 28 anos; no que diz respeito à escolaridade predominou o ensino médio com um quantitativo de 172 (56,4\%) gestantes e quanto ao número de gestações vivenciadas, prevaleceu a frequência entre duas e três gestações, correspondendo à 150 $(49,2 \%)$ do total de gestantes, destaca-se que todos os valores são igualmente significativos $(p<0,001)$, segundo o exposto na Tabela 1.

Tabela 1 - Perfil socioeconômico das usuárias acompanhadas na consulta de pré-natal. Mossoró, RN, Brasil, 2015 (continua)

\begin{tabular}{|c|c|c|}
\hline Variáveis & Freq (\%) & $p$ - valor \\
\hline \multicolumn{3}{|l|}{ Renda } \\
\hline Menos de 1 salário & $66(21,6)$ & $<0,001^{*}$ \\
\hline $1 \mathrm{a} 2$ & $202(66,2)$ & \\
\hline 3 a 5 & $37(12,1)$ & \\
\hline
\end{tabular}




\begin{tabular}{|c|c|c|}
\hline Idade & & \\
\hline 18 a 28 & $190(62,3)$ & $<0,001^{*}$ \\
\hline 29 a 39 & $105(34,4)$ & \\
\hline Acima de 39 & $10(3,3)$ & \\
\hline Média \pm desvio padrão & $26,44 \pm 6,33$ & \\
\hline Mínimo - Máximo & $18-41$ & \\
\hline \multicolumn{3}{|l|}{ Escolaridade } \\
\hline Fundamental & $109(35,7)$ & $<0,001^{*}$ \\
\hline Médio & $172(56,4)$ & \\
\hline Superior & $24(7,9)$ & \\
\hline \multicolumn{3}{|l|}{ Número de gestações } \\
\hline Até 1 & $124(40,7)$ & $<0,001^{*}$ \\
\hline 2 a 3 & $150(49,2)$ & \\
\hline Acima de 3 & $31(10,2)$ & \\
\hline Média \pm desvio padrão & $2,0 \pm 1,22$ & \\
\hline Mínimo - Máximo & $01-11$ & \\
\hline \multicolumn{3}{|c|}{ Participante de programas assistenciais do governo } \\
\hline Sim & $101(33,1)$ & $<0,001^{*}$ \\
\hline Não & $204(66,9)$ & \\
\hline
\end{tabular}

* Significância estatística $(p<0,05)$ - Qui-quadrado para proporções homogêneas

Na infraestrutura, conforme descrição de 25 profissionais, constatou-se que 19 (76\%) edificações foram construídas apropriadamente para as UBS. Em 14 (66\%) unidades não se identificou explicitamente os serviços de pré-natal com descrição de horários, dias e profissionais responsáveis.

No tocante aos suprimentos necessários para as consultas de pré-natal foi identificada a existência de equipamentos indispensáveis (estetoscópio clínico, esfigmomanômetro, fita métrica inelástica, foco de luz, mesa para exames ginecológicos, sonar Doppler, entre outros) (100\%), materiais higiênicossanitários/gestão da informação (fichas para requisição de exames, prescrição de medicamentos, anamnese do pré-natal, encaminhamento para a referência, entre outras; luvas descartáveis, lençóis nas mesas ginecológicas, cartão da gestante, mapa de registro diário, cadastro de gestantes (livro de procedimento), (100\%), $18(72 \%)$ unidades com suprimentos de medicamentos/suplementos e ainda $24(96 \%)$ unidades com apoio laboratorial para exames básicos, nesse quesito foi apresentado um valor significativo $(p<0,001)$, conforme destacado na Tabela 2.

Tabela 2 - Infraestrutura dos serviços da atenção primária de assistência ao pré-natal. Mossoró, RN, Brasil, 2015 (continua)

\begin{tabular}{|c|c|c|}
\hline Variáveis & Freq. $(\%)$ & p-valor \\
\hline \multicolumn{3}{|c|}{ Unidade de saúde em prédio próprio } \\
\hline Adequado & $19(76)$ & $0,009^{*}$ \\
\hline Inadequado & $06(24)$ & \\
\hline \multicolumn{3}{|c|}{ Setor com identificação explícita do pré-natal } \\
\hline Adequado & $11(44)$ & 0,549 \\
\hline Inadequado & $14(66)$ & \\
\hline \multicolumn{3}{|c|}{ Suprimentos de equipamentos } \\
\hline Adequado & $25(100)$ & - \\
\hline Inadequado & 0 & \\
\hline \multicolumn{3}{|c|}{ Suprimento de materiais } \\
\hline Adequado & $25(100)$ & - \\
\hline Inadequado & 0 & \\
\hline
\end{tabular}




\begin{tabular}{lcc}
\hline Existência de medicamentos/suplementos & & \\
\cline { 1 - 2 } Adequado & $0,0028^{*}$ \\
\hline Inadequado & $18(72)$ & $07(28)$ \\
\hline Existência de apoio laboratorial & & \\
\hline Adequado & $24(96)$ & $001^{*}$
\end{tabular}

* Significância estatística ( $p<0,05)$ - Qui-quadrado para proporções homogêneas.

Os dados evidenciam que todas as unidades asseguram os suprimentos necessários para a realização das consultas e acompanhamento pré-natal em relação aos equipamentos indispensáveis e materiais higiênicos-sanitários/gestão da informação e 18 (dezoito) unidades do estudo apresentaram o número adequado de medicamentos/suplementos necessários para assistência de qualidade a gestante.

No que diz respeito as ações realizadas no acompanhamento pré-natal, a Tabela 3 apresenta a percepção dos 25 profissionais e das 305 usuárias.

Tabela 3 - Processo de trabalho da atenção primária de assistência ao pré-natal. Mossoró, RN, Brasil, 2015

\begin{tabular}{|c|c|c|c|c|}
\hline \multirow[t]{2}{*}{ Variáveis } & $\begin{array}{c}\text { Perspectiva } \\
\text { dos } \\
\text { Profissionais } \\
(\mathbf{n}=26) \\
\end{array}$ & $\begin{array}{c}\text { Perspectiva } \\
\text { das } \\
\text { Gestantes } \\
(n=305) \\
\end{array}$ & \multirow[t]{2}{*}{ p-valor $¥$} & \multirow[t]{2}{*}{ p-valor $€$} \\
\hline & Freq. (\%) & Freq. (\%) & & \\
\hline \multicolumn{5}{|c|}{ Realização de Consulta Puerperal } \\
\hline Adequado & $13(37,1)$ & $149(48,9)$ & \multirow[t]{2}{*}{0,128} & \\
\hline Inadequado & $22(62,9)$ & $156(51,1)$ & & 0,689 \\
\hline \multicolumn{5}{|c|}{ Realização de Exames Laboratoriais } \\
\hline Adequado & $26(74,3)$ & $304(99,7)$ & \multirow[t]{2}{*}{$0,004^{*}$} & \\
\hline Inadequado & $09(25,7)$ & $01(0,3)$ & & $<0,001^{*}$ \\
\hline \multicolumn{5}{|c|}{ Realização de Exame de HIV } \\
\hline Adequado & $26(74,3)$ & $304(99,7)$ & \multirow[t]{2}{*}{$0,004^{*}$} & \\
\hline Inadequado & $09(25,7)$ & $01(0,3)$ & & $<0,001^{*}$ \\
\hline \multicolumn{5}{|c|}{ Realização de Imunização Antitetânica } \\
\hline Adequado & $33(94,3)$ & $303(99,3)$ & \multirow[t]{2}{*}{$<0,001^{*}$} & \\
\hline Inadequado & $02(5,7)$ & $02(0,7)$ & & $<0,001^{*}$ \\
\hline \multicolumn{5}{|c|}{ Realização de 6 ou mais consultas durante o Pré-Natal } \\
\hline Adequado & $10(28,6)$ & $195(63,9)$ & \multirow[t]{2}{*}{$0,011^{*}$} & \\
\hline Inadequado & $25(71,4)$ & $110(36,1)$ & & $<0,001^{*}$ \\
\hline \multicolumn{5}{|c|}{ Realização da Primeira consulta de Pré-Natal no $1^{\circ}$ Trimestre de gestação } \\
\hline Adequado & $11(31,4)$ & $240(78,7)$ & \multirow[t]{2}{*}{$0,028^{*}$} & \\
\hline Inadequado & $24(68,6)$ & $65(21,3)$ & & $<0,001^{*}$ \\
\hline \multicolumn{5}{|c|}{ Abordagem multiprofissional } \\
\hline Adequado & $24(68,6)$ & $143(46,9)$ & \multirow[t]{2}{*}{$0,028^{*}$} & \\
\hline Inadequado & $11(31,4)$ & $162(53,1)$ & & $<0,001^{*}$ \\
\hline \multicolumn{5}{|c|}{ Procedimentos clínicos } \\
\hline Adequado & $35(100)$ & $305(100)$ & \multirow[t]{2}{*}{-} & - \\
\hline Inadequado & 0 & 0 & & \\
\hline \multicolumn{5}{|c|}{ Estratégias de educação em Saúde } \\
\hline Adequado & $31(88,6)$ & $186(61)$ & & \\
\hline Inadequado & $04(11,4)$ & 119 (39) & $<0,001^{*}$ & $<0,001^{*}$ \\
\hline
\end{tabular}


*Significância $(p<0,05)$ - Qui-quadrado para proporções homogêneas; ¥p-valores relacionados à perspectivas dos profissionais; $€ \mathrm{p}$-valores referentes à perspectivas das gestantes.

Foi evidenciado que $22(62,9 \%)$, do total de profissionais consideram que não realizam a visita puerperal de modo adequado e $156(51,1 \%)$ usuárias confirmam que não receberam a consulta puerperal. Na realização dos exames laboratoriais, 26 (74,3\%) profissionais afirmam ser adequado, aspecto endossado por $304(99,7 \%)$ usuárias ( $p<0,001)$. Quanto ao exame de HIV, $26(74,3 \%)$ profissionais avaliam a realização como adequada, confirmado por $304(99,7 \%)$ usuárias $(p<0,001)$. Na realização de imunização antitetânica, 33 (94,3\%) profissionais afirmaram ser adequada, fato evidenciado por 303 $(99,3 \%)$ usuárias imunizadas quase em sua totalidade, nesse aspecto os valores são respectivamente significativos $(p<0,001)$.

Entretanto, há divergências no número de consultas durante o pré-natal. Tomando por base o preconizado pelo Ministério da Saúde, 25 (71,4\%) profissionais afirmam que há uma discordância, já 195 (63,9\%) usuárias referem que o número de consultas realizadas durante o pré-natal foi apropriado. Percebe-se o mesmo impasse na realização da primeira consulta de pré-natal a partir do primeiro trimestre gestacional, quando $24(68,6 \%)$ profissionais consideram ineficaz a captação precoce, em contrapartida $240(78,7 \%)$ usuárias afirmam ter realizado adequadamente.

No que diz respeito à abordagem multiprofissional, 162 (53,1\%) usuárias referiram não terem sido acompanhadas por mais de um profissional de saúde durante a consulta de pré-natal, dado divergente do discurso de $24(68,6 \%)$ profissionais que consideram adequada. Os profissionais em sua totalidade realizavam os procedimentos clínico-obstétricos mínimos considerados necessários para o pré-natal e $31(88,6 \%)$ profissionais efetivavam pelo menos uma atividade de educação em saúde, extra consultas, dados evidenciados por 186 (61\%) usuárias ao confirmarem a participação nessas atividades.

\section{DISCUSSÃO}

Observou-se que o perfil socioeconômico das usuárias em pré-natal apresentou-se semelhante a outros estudos realizados, com predomínio de gestantes adultas jovens, com baixa renda familiar e escolaridade oscilando entre ensino médio e fundamental. Isso tende a contribuir para que essas mulheres tenham mais acesso a empregos com baixas remunerações ${ }^{(12-13)}$.

Quanto aos locais de instalação das UBS, há estudos que apresentam as mesmas peculiaridades que alguns profissionais das UB citaram, no que diz respeito a estruturação física desses espaços. Muitas unidades são instaladas em prédios improvisados, existentes na comunidade e não apresentam construção específica para atender as necessidades de todos os usuários e mesmo dos profissionais que lá trabalham ${ }^{(14)}$.

Conforme os manuais, a estrutura física deve contemplar ventilação adequada e privacidade para as consultas, além de permitir manutenção da higiene, atendimento inicial e transporte seguro em casos de urgência ${ }^{(2)}$.

A falta de estrutura adequada e de instalações próprias para o cuidado assistencial compromete significativamente a promoção da saúde das gestantes e dos usuários em geral. Tal condição tende a desqualificar a unidade quanto à humanização do atendimento e remete a precariedade nas condições de trabalho relativas às instalações físicas e a disponibilidade e manutenção de equipamentos ${ }^{(10)}$.

Para que a qualidade e a eficiência estejam presentes na assistência pré-natal, é indispensável, inclusive,um ambiente com infraestrutura apropriada, pois práticas adequadas requerem a existência ambiente de trabalho que suporte a excelência do desempenho ${ }^{(15)}$.

O suprimento de material de consumo, os equipamentos e os medicamentos receberam avaliações positivas dos profissionais. Estes são importantes instrumentos de trabalho, sendo assim, a insuficiência desses recursos materiais compromete o desenvolvimento e a qualidade da assistência prestada ${ }^{(16)}$.

$\mathrm{Na}$ maioria das unidades o apoio laboratorial foi evidenciado como positivo, com a garantia da realização dos exames recomendados para cada período gestacional. A realização destes contribui para 
o diagnóstico e/ou a prevenção de agravos/doenças como anemias, sífilis congênita, AIDS, infecção urinária, entre outras, sendo capazes de reduzir riscos de aquisição de morbidades e mortalidade perinatal e neonatal ${ }^{(17)}$.

Além da dimensão estrutural enquanto interveniente ao processo, a dinâmica de interação, e, mais ainda, os cuidados ofertados pela equipe de saúde, ou seja, o processo de trabalho cotidiano,são indispensáveis para a qualificação da assistência. O processo de trabalho se refere às ações desenvolvidas nos serviços de saúde, orientadas essencialmente para a análise da atuação profissional no cuidado do binômio saúde/doença da população. Avaliar o processo requer critérios que são delimitados, geralmente, pela resolutividade das ações desenvolvidas ${ }^{(18)}$.

No que diz respeito ao número de consultas pré-natais, esse quantitativo deve se constituir como ferramenta para a elaboração de protocolos assistenciais e planejamento dos recursos em saúde ${ }^{(8)}$. Teoricamente, um número maior de consultas pode significar mais oportunidades de cuidados preventivos e de promoção de saúde, especialmente em gestações de maior risco, com maior probabilidade de interferir nos desfechos perinatais ${ }^{(19)}$.

Para o índice IPR-PRÉNATAL, o número de consultas adequado é $\geq 6$ consultas, porém esse número não é prevalente entre as unidades do município de Mossoró/RN. Nesse quesito, o Ministério da Saúde (MS) reforça que, ainda que haja um número reduzido de consultas, quando se é dada maior ênfase para o conteúdo de cada uma delas, não há aumento de intercorrências nos resultados perinatais ${ }^{(19)}$.

Os cuidados no primeiro trimestre são usados como o maior indicador da qualidade dos cuidados maternos. Portanto, o calendário deve ser iniciado o mais precocemente, ou seja, no primeiro trimestre, e deve ser regular, garantindo-se que todas as ações propostas sejam efetivadas ${ }^{(19)}$.

Estudos que avaliam o pré-natal mostraram resultados positivos, quando trazem em seus dados que mais de $50 \%$ das gestantes haviam iniciado as consultas no primeiro trimestre ${ }^{(20)}$, esses resultados apontados distanciam-se dos dados desse estudo. Nesse sentido, percebe-se um desacordo entre os dados apontados na pesquisa e os demais estudos.

Ainda sobre a dinâmica de consultas, o índice IPR/PN(9) considera uma abordagem multiprofissional adequada, quando durante o acompanhamento existe a presença de mais de um profissional de nível superior na assistência. Essa variável do estudo mostrou que uma parcela considerável das usuárias entrevistadas teve acesso a uma abordagem multiprofissional durante seu acompanhamento pré-natal. A equipe multidisciplinar dá suporte, procurando entender o meio em que vive a paciente, levando em conta os aspectos sócio econômicos, culturais e religiosos, auxiliando no desenvolvimento da cidadania $^{(21)}$.

Daí a importância do envolvimento desses profissionais, no sentido de adentrar a realidade das gestantes que realizam o pré-natal, utilizar e articular o conhecimento técnico-científico, na tentativa de intervir de modo a contribuir para uma gestação que evolua sem intercorrência.

É imprescindível compreender que uma atenção de qualidade não é alcançada através de um cuidado pautado nas ações de um único profissional, é necessário desenvolver uma linha assistencial que contemple a participação de toda a equipe de saúde, sendo elaborada de maneira coletiva, para que, de fato, ocorra a integralidade dos indivíduos ${ }^{(22)}$.

No intercurso do acompanhamento, algumas questões precisam ser enfatizadas na perspectiva de contribuir para a minimização dos riscos e agravos à saúde da mãe e do concepto, entre elas a realização de exames e das vacinas. Destaca-se a importância não somente da marcação e solicitação, mas da realização destes no período gestacional, pois a vacinação e os exames durante a gestação objetiva, não somente a proteção da gestante, como também do feto.

A realização das vacinas nos prazos delimitados, com resultados positivos nesta pesquisa para a Imunização Antitetânica, tende a prevenir possíveis intercorrências e agravos durante a gestação. Estudos apontam que a realização dos exames, adequados segundo os profissionais para HIV, é um componente do pré-natal e a ausência dos resultados para as gestantes representa a perda de oportunidade de diagnóstico e tratamento de agravos passíveis de controle ${ }^{(18,23)}$.

Outra dimensão que merece ser destacada é a consulta puerperal, esta deve ser realizada na primeira 
semana após a alta do recém-nascido. Na oportunidade serão avaliados o estado de saúde da mulher e do bebê, a interação mãe/recém-nascido, identificação de situações de risco ou intercorrências e condutas a serem implementadas, bem como orientação sobre os cuidados básicos com o recémnascido, planejamento familiar e apoio a família para a amamentação ${ }^{(18)}$.

Partindo dos dados apresentados, observou-se que muitos profissionais de saúde não conseguem realizar a consulta puerperal e isso reflete na qualidade da assistência. Outros estudos corroboram esse achado, quando se afirma que dentre as atividades desenvolvidas durante o programa de pré-natal, a consulta puerperal mostra-se deficitária ${ }^{(24)}$. Assim, cabe repensar essa prática no âmbito da atenção básica, uma vez que durante a consulta, além das orientações supracitadas, podem ser identificadas situações de risco para o bebê e para a puérpera ${ }^{(19)}$.

Os dados evidenciam que a maioria dos profissionais executa algum tipo de atividade de educação em saúde durante o acompanhamento à gestante e as usuárias reforçam esses dados. Os documentos oficiais do MS sobre a assistência à mulher sugerem, normatizam e estabelecem a educação em saúde no pré-natal. Eles estão respaldados por estudos científicos que comprovam sua efetividade na promoção da saúde durante o evento reprodutivo e dá subsídios para a continuidade da implementação das ações educativas no âmbito do SUS ${ }^{(19)}$.

Para o desenvolvimento de uma assistência pré-natal qualificada e humanizada é necessário pensar nas dimensões que interatuam nesse cuidado, como a infraestrutura dos serviços de saúde e o processo de trabalho. Essas dimensões são indispensáveis para concretização de um cuidado integral, pois a infraestrutura atua como uma ferramenta facilitadora do processo de trabalho, favorecendo a otimização de recursos e o atendimento humanizado, acolhedor e resolutivo. O processo de trabalho quando pautado na humanização da assistência, valoriza o vínculo com a gestante, como também a efetivação de ações de educação em saúde, abordagem multiprofissional que garantem um olhar diferenciado sobre a usuária, na intenção de atender as suas necessidades.

\section{CONCLUSÃO}

Os resultados deste estudo, embora representem uma realidade específica, apresentam dados importantes relacionados a situação da assistência ao pré-natal na atenção básica de um município do Rio Grande do Norte.

Foi visualizado que $82 \%$ das unidades básicas apresentou adequada relação a quase todas as variáveis do componente infraestrutura, sendo esse resultado favorável para proporcionar atendimento de qualidade e humanizado, entendendo que é respeitável oferecer ambiente que acolha as gestantes e ofereça conforto e privacidade no momento da consulta.

Ao avaliar o processo de trabalho, destacou-se o resultado referente a consulta puerperal. Essa atividade não é executada pela maioria dos profissionais, fazendo com que a puérpera não receba o cuidado pós-parto, o que pode determinar riscos para saúde da mãe e do recém-nascido. O número de consultas realizadas durante o acompanhamento pré-natal também foi considerado como inadequado, de acordo com os profissionais. Porém, no ponto de vista das gestantes, o número de consultas foi realizado adequadamente, o que estimula a reflexão acerca das práticas e das necessidades ora percebidas pelos diferentes atores envolvidos nesse processo.

Percebe-se que muitos entraves podem ser decorrentes de diversos aspectos que limitam a aplicação das normas ministeriais pelos serviços de saúde, sejam eles relacionados ao desconhecimento e despreparo dos profissionais para atuação na atenção básica, ou pelo próprio contexto político, econômico e social que comanda a produção das práticas nos serviços de saúde.

Entende-se que apesar dos apontamentos e das soluções possíveis mediante resultados desse tipo de estudo, sabe-se que nem todos os problemas evidenciados terão resolutividade no âmbito local e no plano de ação pessoal. Nesse sentido, ressalta-se a importância da elaboração de estudos acerca da avaliação do pré-natal de baixo risco, uma vez que a partir dessa avaliação, sugestões e objetivos poderão ser elaborados e ações implementadas, no intuito de propiciar a melhor assistência realizada durante o pré-natal. 
1. Zanoteli S, Zatti CA, Ferraboli SF Intercorrências clínicas da gestação. Braz. J. Surg. Clin. Res. [Internet] 2013;4(2) [acesso em 14 jan 2015]. Disponível: http://www.mastereditora.com.br/periodico/20130831_181026.pdf.

2. Ministério da Saúde (BR). Secretaria de Atenção à Saúde. Departamento de Ações Programáticas Estratégicas. Gestação de alto risco: manual técnico. $5^{\text {a }}$ ed. Brasília: Ministério da Saúde; 2010.

3. Ministério da Saúde (BR). Pré-natal e Puerpério: atenção qualificada e humanizada: Manual técnico. $3^{\text {a }}$ ed. Brasília: Ministério da Saúde; 2006.

4. Kassebaum NJ, Bertozzi-Villa A, Coggeshal MS, Shackelford KA, Steiner C, Heuton KR, et al. Global, regional, and national levels and causes of maternal mortality during 1990-2013: a systematic analysis for the Global Burden of Disease Study 2013. Lancet. [Internet] 2014;384(9947) [acesso em 10 dez 2014]. Disponível: http:// dx.doi.org/10.1016/S0140-6736(14)60696-6.

5. Ministério da Saúde (BR). Sistema de Informações sobre Mortalidade - SIM. Painel de Monitoramento da Mortalidade Materna. [Internet] Brasília: Ministério da Saúde; 2014 [acesso em 30 out 2014]. Disponível: http:// svs.aids.gov.br/dashboard/mortalidade/materna.show.mtw.

6. Ministério da Saúde (BR). Secretaria de Atenção à Saúde. Departamento de Atenção Básica. Avaliação normativa do Programa Saúde da Família no Brasil: monitoramento da implantação e funcionamento das equipes de saúde da família: 2001-2002. Brasília: Ministério da Saúde; 2004.

7. Sword W, Heaman M, Biro MA, Homer C, Yelland J, Akhtar-Danesh N, et al. Quality of prenatal care questionnaire: psychometric testing in an Australia population. BMC Pregnancy Childbirth. [Internet] 2015;(15) [acesso em 10 dez 2014]. Disponível: http://dx.doi.org/10.1186/s12884-015-0644-7.

8. Anversa ETR, Bastos GAN, Nunes LN, Dal Pizzol TS. Qualidade do processo da assistência pré-natal: unidades básicas de saúde e unidades de Estratégia Saúde da Família em município no Sul do Brasil. Cad. Saúde Pública. [Internet] 2012;28(4) [acesso em 23 nov 2014]. Disponível: http://dx.doi.org/10.1590/S0102-311X2012000400018.

09. Ministério da Saúde (BR). Projeto de avaliação nacional do Programa de Humanização do Pré-natal e Nascimento (PHPN). Brasília: Ministério da Saúde; 2004.

10. Silva EP. Proposta de um índice para avaliação da assistência pré-natal na atenção básica [dissertação]. João Pessoa (PB): Universidade Federal da Paraíba; 2012.

11. Donabedian A. The quality of care: How can it be assessed?.JAMA. [Internet] 1988;260(12) [acesso em 14 jan 2016]. Disponível: http://post.queensu.ca/ hh11/assets/applets/The_Quality_of_Care__How_Can_it_Be_ Assessed_-_Donabedian.pdf.

12. Pimenta AM, Nazareth JV, de Souza KV, PimentaGM.Programa "Casa das Gestantes": perfil das usuárias e resultados da assistência à saúde materna e perinatal. Texto Contexto Enferm. [Internet] 2012;21(4) [acesso em 23 nov 2014] Disponível: http://dx.doi.org/10.1590/S0104-07072012000400023.

13. Leite FMC, Barbosa TKO, Mota JS, Nascimento LCN, Amorim MHC, Primo CC. Perfil socioeconômico e obstétrico de puérperas assistidas em uma maternidade filantrópica. Cogitare Enferm. [Internet] 2013;18(2) [acesso em 25 abr 2016] Disponível: http://dx.doi.org/10.5380/ce.v18i2.32584.

14. Facchini LA, Piccini RX, Tomasi E, Thumé E, Silveira DS, Siqueira FV, et al. Desempenho do PSF no Sul e no Nordeste do Brasil: avaliação institucional e epidemiológica da Atenção Básica à Saúde. Ciênc. saúde coletiva. [Internet] 2006;11(3)[acesso em 03 fev 2015] Disponível: http://dx.doi.org/10.1590/S1413-81232006000300015.

15. da Silveira DS, dos Santos IS, da Costa JSD. Atenção pré-natal na rede básica: uma avaliação de estrutura e do processo. Cad. saúde pública. [Internet] 2001;17(1)[acesso em 14 out 2014] Disponível: http://dx.doi.org/10.1590/ S0102-311X2001000100013.

16. Conselho Internacional de Enfermeiros (CME). Ambientes favoráveis à prática: Condições no trabalho, cuidados de qualidade. [Internet] 2007 [acesso em 04 mar 2015]. Disponível: http://www.ordemenfermeiros.pt/ publicacoes/Documents/Kit_DIE_2007.pdf. 
17. Ministério da Saúde (BR). Programa humanização do parto: humanização no pré-natal e nascimento. Brasília: Ministério da Saúde; 2004.

18. Ximenes Neto FRG, Leite JL, Fuly PSC, Cunha ICKO, Clemente AS, Dias MSA, et al. Qualidade da atenção ao pré-natal na Estratégia Saúde da Família em Sobral, Ceará. Rev. bras. enferm. [Internet] 2008;61(5)[acesso em 14 jan 2015]. Disponível: http://dx.doi.org/10.1590/S0034-71672008000500011.

19. Domingues RMSM, Hartz ZMA, Dias MAB, Leal MC. Avaliação da adequação da assistência pré-natal na rede SUS do Município do Rio de Janeiro, Brasil. Cad. Saúde Pública. [Internet] 2012;28(3) [acesso em 10 dez 2014$].$ Disponível: http://dx.doi.org/10.1590/S0102-311X2012000300003.

20. Ministério da Saúde (BR). Secretaria de Atenção à Saúde. Departamento de Atenção Básica. Atenção ao prénatal de baixo risco. Brasília: Ministério da Saúde; 2013.

21. Zanchi M, Gonçalves CV, Cesar JA, Dumith SC. Concordância entre informações do Cartão da Gestante e do recordatório materno entre puérperas de uma cidade brasileira de médio porte. Cad. Saúde Pública. [Internet] 2013;29(5) [acesso em 04 mar 2015]. Disponível: http://dx.doi.org/10.1590/S0102-311X2013000500019.

22. Matos DS, Rodrigues MS, Rodrigues TS. Atuação do enfermeiro na assistência ao pré-natal de baixo risco na estratégia saúde da família em um município de Minas Gerais. Rev. Enfermagem Revista. [Internet] 2013;16(1) [acesso em 22 nov 2014]. Disponível: http://periodicos.pucminas.br/index.php/enfermagemrevista/article/ view/5282/5237.

23. Grando T, Cardoso SMM, Fontana R, Rodrigues FCP. Consulta pré-natal: satisfação das usuárias do sus. Cogitare Enferm.[Internet] 2012;17(2) [acesso em 25abr 2016]. Disponível: http://dx.doi.org/10.5380/ce.v17i2.25565.

24. Zampieri MFM, Erdmann AL. Cuidado humanizado no pré-natal: um olhar para além das divergências e convergências. Rev. Bras. Saúde Matern. Infant. [Internet] 2010;10(3) [acesso em 12 fev 2015]. Disponível: http:// dx.doi.org/10.1590/S1519-38292010000300009. 